\title{
Hospital readmissions after pulmonary resection: post-discharge nursing telephone assessment identifies high risk patients
}

\author{
Robert M. Van Haren ${ }^{1}$, Arlene M. Correa ${ }^{2}$, Boris Sepesi ${ }^{2}$, David C. Rice ${ }^{2}$, Wayne L. Hofstetter ${ }^{2}$, \\ Jack A. Roth ${ }^{2}$, Stephen G. Swisher ${ }^{2}$, Garrett L. Walsh ${ }^{2}$, Ara A. Vaporciyan ${ }^{2}$, Reza J. Mehran ${ }^{2}$, \\ Mara B. Antonoff ${ }^{2}$
}

${ }^{1}$ Division of Thoracic Surgery, Department of Surgery, University of Cincinnati College of Medicine. Cincinnati, OH, USA; ${ }^{2}$ Department of Thoracic and Cardiovascular Surgery, The University of Texas M.D. Anderson Cancer Center, Houston, TX, USA

Contributions: (I) Conception and design: RM Van Haren, MB Antonoff; (II) Administrative support: RM Van Haren, AM Correa, MB Antonoff; (III) Provision of study materials or patients: All authors; (IV) Collection and assembly of data: RM Van Haren, AM Correa; (V) Data analysis and interpretation: All authors; (VI) Manuscript writing: All authors; (VII) Final approval of manuscript: All authors.

Correspondence to: Mara B. Antonoff, MD. Department of Thoracic and Cardiovascular Surgery, The University of Texas M.D. Anderson Cancer Center, 1400 Pressler St, Unit 1489, Houston, TX 77030, USA. Email: mbantonoff@mdanderson.org.

Background: We previously reported that post-discharge nursing telephone assessments identified a frequent number of patient complaints. Our aim was to determine if telephone assessments can identify patients at risk for emergency room (ER) visits or hospital readmissions.

Methods: A single-institution, retrospective review was performed on all patients undergoing pulmonary resection over a 12-month period. Standardized nursing telephone calls were conducted and records were reviewed to determine postoperative issues. ER visits and readmissions within 30 and 90 days were recorded. Results: In total, 521 patients underwent pulmonary resection and 245 (47\%) were reached for telephone assessment. ER visits within 30/90 days were 8.1\% ( $n=42)$ and $12.1 \%(n=63)$. Readmissions within 30/90 days were $3.1 \%(n=16)$ and $6 \%(n=31)$. For those reached by telephone assessment, patients with major issue demonstrated increased 30-day ER visits: $22.6 \%(\mathrm{n}=7)$ vs. $8.0 \%(\mathrm{n}=17), \mathrm{P}=0.019$. For all patients, those with 90-day ER visit and/or readmission were more likely to have pulmonary complications during initial admission $(43.8 \%$ vs. $21.2 \%, \mathrm{P}<0.001)$. Among patients who were reached by telephone, independent predictors of ER visit or readmission within 30 days were: major issue identified on telephone assessment $(\mathrm{P}=0.007)$, discharge with chest tube $(<0.001)$, and reintubation postoperatively $(\mathrm{P}=0.047)$.

Conclusions: Standardized nursing telephone assessments were able to identify a high-risk population more likely to need ER visit or readmission. However, telephone assessments did not decrease ER visits or readmissions. Improved post-discharge protocols are needed for these high-risk patients in order to ensure patient safety, optimize patient experience, and limit unnecessary resource utilization.

Keywords: Readmission; pulmonary resection; nursing telephone assessment

Submitted Dec 30, 2019. Accepted for publication Feb 06, 2020.

doi: $10.21037 /$ jtd.2020.02.08

View this article at: http://dx.doi.org/10.21037/jtd.2020.02.08 


\section{Introduction}

Unplanned hospital readmissions after surgery negatively impact patients and are associated with increased mortality $(1,2)$. A substantial cost to the healthcare system is also created by hospital readmissions (3-5). Hospital readmissions serve as a quality metric by which hospitals and providers are judged (6). After pulmonary resection, hospital readmissions are estimated to be $7-12 \%$ at 30 days and as high as $20 \%$ at 90 days (7-10). Risk factors for readmission after pulmonary resection include longer operative times, prolonged air leak, decreased predicted forced expiratory volume in 1 second $\left(\mathrm{FEV}_{1}\right)$, and discharge to nursing home facility (11).

There is considerable interest in identifying predictors of hospital readmission and implementing programs to decrease its occurrence. In 2012, the Hospital Readmission Reduction Program was introduced by the Centers for Medicare and Medicaid Services (CMS). This program reduces reimbursement to hospital with increased readmissions for acute myocardial infarction, chronic obstructive pulmonary disease, heart failure, pneumonia, coronary artery bypass graft surgery, and elective total hip arthroplasty/total knee arthroplasty $(12,13)$.

Post-discharge nursing telephone assessments can evaluate patient complaints, offer reassurance and instructions, and monitor recovery after procedures (14-18). We previously reported that post-discharge nursing telephone assessments identified a frequent number of patient complaints after discharge from pulmonary resection (19). The aim of this study was to determine if post-discharge nursing telephone assessments can identify patients who are high risk for emergency room (ER) visits or hospital readmissions.

\section{Methods}

After obtaining approval from the institutional review board (PA14-0771), a retrospective review was performed on consecutive patients undergoing pulmonary resection at the University of Texas MD Anderson Cancer Center. We included patients with primary lung cancer and as well as pulmonary metastatic disease. The study period was 12 months: April 2013-March 2014.

As previously described (19), our group implemented a standardized post-discharge patient phone call in 2013. Clinical Nurse Leaders on the thoracic surgery inpatient floor were tasked with completing the phone calls. Patients were contacted and asked specific questions to identify any postoperative issues or complaints, including: diet, appetite, pain, medication usage, bowel activity, difficulty breathing, physical activity level, social support, and characteristics of surgical wound. Any additional concerns from the patient or their primary caregiver were also documented. The Clinical Nurse Leader completed a standardized note in the electronic medical record. Postoperative issues and concerns were addressed following our developed protocol. No intervention was performed for patients without any issues identified. The Clinical Nurse Leader provided counseling for minor issues such as correct medication dosage, routine wound care, and questions about activity restrictions. Patients with major issues were triaged as previously described (19) with appropriate referrals made to inpatient practitioners for follow up, clinic appointments scheduled, and/or ER referral.

Data were obtained from our prospectively maintained departmental database, including patient demographics, medical comorbidities, operative details such as approach and extent of resection, and postoperative events. Medical records were reviewed to determine ER visits and readmissions within 30 and 90 days.

Statistical analyses were performed with SPSS Ver. 21.0 (IBM Corporation, Armonk, NY). Student's $t$-test compared independent data. $\chi^{2}$ test and Fisher's exact test was used to compare categorical data. Multiple logistic regression was performed and covariates with $\mathrm{P}<0.25$ were entered into multivariable analysis. Stepwise Wald backward elimination was used in multivariable logistic regression analysis to identify variables independently associated with the ER visit and hospital readmissions. Values are expressed as mean \pm standard deviation. Significance was assessed at $\mathrm{P}<0.05$.

\section{Results}

During the 12-month study period, 523 patients underwent pulmonary resection and 521 patients survived to hospital discharge. The median age was $60 \pm 15$ years and most were male $(n=271,52 \%)$. The most common extent of resection was sublobar $(\mathrm{n}=259,50 \%)$, followed by lobectomy ( $\mathrm{n}=232,44 \%)$. The most frequent operative approach was thoracotomy $(\mathrm{n}=363,70 \%)$, with videoassisted thoracoscopic surgery comprising $26 \%(\mathrm{n}=134)$ and robotic-assisted thoracoscopic surgery $6 \%(\mathrm{n}=29)$.

Two hundred and forty-five patients (47\%) were reached 
Table 1 Univariate analysis between patients with 30-day ER visit or hospital readmission $v s$. none, all patients

\begin{tabular}{|c|c|c|c|}
\hline Variables & $\begin{array}{l}\text { No ER visit/ } \\
\text { readmission } \\
\quad(n=457)\end{array}$ & $\begin{array}{l}\text { ER visit/ } \\
\text { readmission } \\
(n=64)\end{array}$ & $P$ \\
\hline Age, years & $59 \pm 15$ & $58 \pm 12$ & 0.870 \\
\hline Sex, male & $52 \%$ & $55 \%$ & 0.726 \\
\hline Race & & & 0.990 \\
\hline Caucasian & $80 \%$ & $82 \%$ & \\
\hline African American & $8 \%$ & $7 \%$ & \\
\hline Local residence & $27 \%$ & $50 \%$ & 0.001 \\
\hline Extent of surgery & & & 0.172 \\
\hline Sublobar & $51 \%$ & $34 \%$ & \\
\hline Lobectomy & $43 \%$ & $57 \%$ & \\
\hline Bilobectomy & $2 \%$ & $2 \%$ & \\
\hline Pneumonectomy & $4 \%$ & $7 \%$ & \\
\hline $\begin{array}{l}\text { Post discharge telephone } \\
\text { call }\end{array}$ & $46 \%$ & $56 \%$ & 0.174 \\
\hline Approach & & & 0.316 \\
\hline Thoracotomy & $69 \%$ & $80 \%$ & \\
\hline VATS & $26 \%$ & $18 \%$ & \\
\hline Robotic & $5 \%$ & $2.3 \%$ & \\
\hline Pulmonary complications & $21 \%$ & $53 \%$ & $<0.001$ \\
\hline Discharge with chest tube & $7 \%$ & $40 \%$ & $<0.001$ \\
\hline Discharge on oxygen & $8 \%$ & $6 \%$ & 0.752 \\
\hline
\end{tabular}

for telephone assessment, while $276(53 \%)$ were not able to be contacted. The demographics of whose individuals who were reached for telephone assessment and those not reached were not significantly different (data not shown).

Among all patients, ER visits within 30 and 90 days were $8.1 \%(\mathrm{n}=42)$ and $12.1 \%(\mathrm{n}=63)$, respectively. Readmissions within 30 and 90 days were $3.1 \%(n=16)$ and $6 \%(n=31)$. Likelihood of ER visit or readmission was not dependent on whether patients were reached for telephone assessment $(\mathrm{P}=0.523)$. For all patients, those with 30 -day ER visit or readmission were more likely to have pulmonary complications during initial admission $(\mathrm{P}<0.001)$, including discharge with chest tube $(\mathrm{P}<0.001)$ (Table 1). Patients with 90-day ER visit or readmission were also more likely to have pulmonary complications during initial admission
Table 2 Univariate analysis between patients with 90-day ER visit readmission $v s$. none, all patients

\begin{tabular}{|c|c|c|c|}
\hline Variables & $\begin{array}{l}\text { No ER visit/ } \\
\text { readmission } \\
\quad(n=457)\end{array}$ & $\begin{array}{l}\text { ER visit/ } \\
\text { readmission } \\
\quad(n=64)\end{array}$ & $P$ \\
\hline Age, years & $59 \pm 15$ & $61 \pm 11$ & 0.388 \\
\hline Sex, male & $52 \%$ & $53 \%$ & 0.850 \\
\hline Race & & & 0.901 \\
\hline Caucasian & $80 \%$ & $84 \%$ & \\
\hline African American & $8 \%$ & $6 \%$ & \\
\hline Local residence & $27 \%$ & $44 \%$ & 0.005 \\
\hline Extent of surgery & & & 0.060 \\
\hline Sublobar & $52 \%$ & $36 \%$ & \\
\hline Lobectomy & $43 \%$ & $53 \%$ & \\
\hline Bilobectomy & $2 \%$ & $3 \%$ & \\
\hline Pneumonectomy & $4 \%$ & $8 \%$ & \\
\hline $\begin{array}{l}\text { Post discharge telephone } \\
\text { call }\end{array}$ & $46 \%$ & $52 \%$ & 0.437 \\
\hline Approach & & & 0.218 \\
\hline Thoracotomy & $69 \%$ & $78 \%$ & \\
\hline VATS & $27 \%$ & $20 \%$ & \\
\hline Robotic & $5 \%$ & $2 \%$ & \\
\hline Pulmonary complications & $21 \%$ & $44 \%$ & $<0.001$ \\
\hline Discharge with chest tube & $7 \%$ & $33 \%$ & $<0.001$ \\
\hline Discharge on oxygen & $8 \%$ & $6 \%$ & 0.570 \\
\hline
\end{tabular}

$(\mathrm{P}<0.001)$, including discharge with chest tube $33 \%$ vs. $7 \%$, $\mathrm{P}<0.001$ ) (Table 2). Patient with $\mathrm{ER}$ visit or readmission at 30 or 90 days were more likely to be local resident (within 50 miles of medical center $)(\mathrm{P}=0.001$ and $\mathrm{P}=0.005)$.

Next, subgroup analysis was performed on those reached for telephone assessment $(n=245)$. Patients identified to have major issues on telephone assessment demonstrated increased 30-day ER visits: $22.6 \%(\mathrm{n}=7)$ vs. $8.0 \% \quad(\mathrm{n}=17)$, $\mathrm{P}=0.019$ and trended toward increased 30-day readmission $9.7 \%(\mathrm{n}=3)$ vs. $2.3 \%(\mathrm{n}=5), \mathrm{P}=0.066$ (Figure 1 ).

Multiple logistic regression was performed on the composite outcome of ER visit or readmission. For all patients, pulmonary complications were independent associated with ER visits or hospital readmissions at 30 and 90 days (Table 3). Among the subgroup reached for 


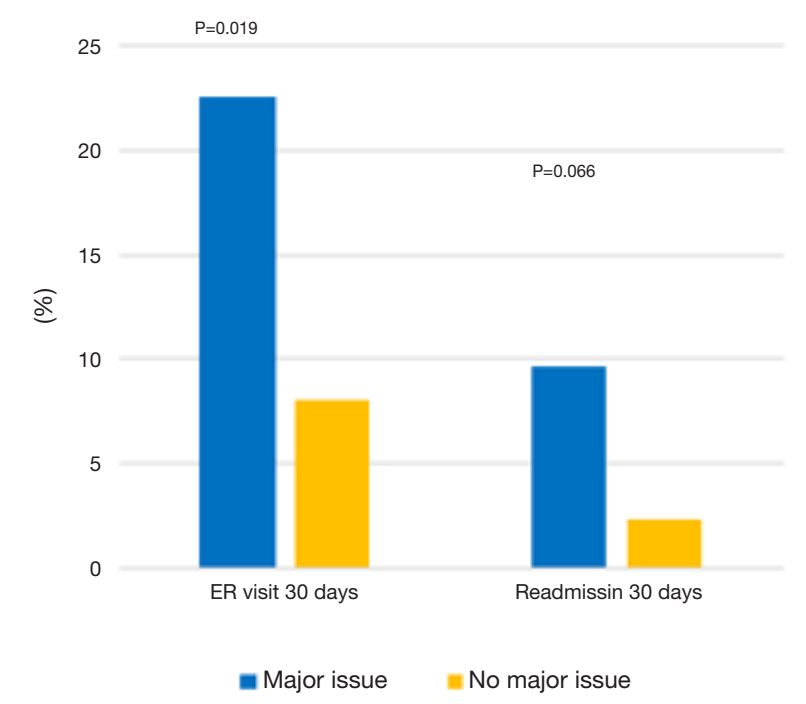

Figure 1 Thirty-day ER visit and readmission rate between patients with major issues identified on telephone assessment and those with no major issue. ER, emergency room.

Table 3 Logistic regression for 30 and 90 ER visit or readmission, all patients

\begin{tabular}{lllll}
\hline Outcomes and variables & P value & OR & \multicolumn{2}{c}{$95 \% \mathrm{Cl}$ for OR } \\
\cline { 4 - 5 } & & & Lower & Upper \\
\hline $\begin{array}{l}\text { ER visit or readmit } 30 \text { days, } \\
\text { all-patients } \mathrm{n}=521\end{array}$ & & & & \\
$\quad$ Pulmonary complications & $<0.001$ & 4.027 & 2.143 & 7.566 \\
ER visit or readmit 90 days, & & & & \\
all-patients $\mathrm{n}=521$ & & & & \\
$\quad$ Pulmonary complications & $<0.001$ & 2.917 & 1.695 & 5.019 \\
\hline
\end{tabular}

telephone assessment, logistic regression identified: major issue on telephone assessment $(\mathrm{P}=0.007)$, discharge with chest tube $(\mathrm{P}<0.001)$, and reintubation postoperatively $(\mathrm{P}=0.048)$ as independently associated with $\mathrm{ER}$ visit or readmission within 30 days (Table 4). Variables independently associated with $\mathrm{ER}$ visit or readmission within 90 days were length of stay $(\mathrm{P}=0.038)$ and discharge with chest tube $(\mathrm{P}=0.001)$ (Table 4).

\section{Discussion}

In our study, we identified a significant number of ER visits at 30 and 90 days: $8.1 \%(\mathrm{n}=42)$ and $12.1 \%(\mathrm{n}=63)$, and hospital readmission at 30 and 90 days: $3.1 \%(n=16)$ and $6 \%(\mathrm{n}=31)$. Inpatient pulmonary complications were independently associated with ER visit and hospital readmission. Standardized nursing telephone assessments were able to identify a high-risk population likely to need ER visit or readmission. However, additional protocols are needed to decrease readmission in this group of patients.

Readmission after pulmonary resection was relatively low in our study, but other studies have reported higher rates. A review of the American College of Surgeons National Surgical Quality Improvement Program database demonstrated a 30 -day readmission rate of $9.3 \%$ for patients undergoing pulmonary resection for any indication. There was no difference in readmission rates between open lobectomy and video-assisted thoracoscopic lobectomy. Pulmonary complications during the initial hospitalization were strongly associated with hospital readmission (7). Hu et al. (1) analyzed the Surveillance Epidemiology and End Results (SEER)-Medicare registry from 2006-2011 for patients undergoing lung cancer resection and found the readmission rate was $12.8 \%$. Readmission was related to pulmonary complications such as pneumonia, respiratory insufficiency, pneumothorax, and cardiac events. Most importantly, 90-day mortality was increased by 6 -fold after hospital readmission.

We previously reported that post-discharge nursing telephone assessments identified a frequent number of patient complaints after discharge from pulmonary resection (19). Issues identified included constipation, shortness of breath, and inadequate pain control. Most issues were minor and addressed on the telephone assessment, however $13 \%$ required escalation of care (19). In this study, we found that major complaints identified on telephone assessment were independently associated with ER visit and hospital readmission. Previous studies analyzing nursing post-discharge telephone assessment after medical admission have demonstrated reduced hospital readmissions for those who received telephone call (15). However, a recent Cochrane review of 33 studies concluded there was not sufficient evidence to conclude that telephone assessments were an effective intervention (16).

In our study, post discharge nursing telephone assessments were able to identify patient high risk for ER visit or readmission, however telephone assessments alone were not sufficient to decreases readmissions. There is a need for improved post discharge protocols to prevent 
Table 4 Logistic regression for 30 and 90 ER visit or readmission, patients who received telephone assessment

\begin{tabular}{|c|c|c|c|c|}
\hline Outcomes and variables & $P$ value & OR & \multicolumn{2}{|c|}{$95 \% \mathrm{Cl}$ for OR } \\
\hline \multicolumn{5}{|c|}{ ER visit or readmit 30 days, patients who received telephone assessment $(n=245)$} \\
\hline Discharge with chest tube & $<0.001$ & 9.391 & 3.237 & 27.245 \\
\hline Reintubation post-op & 0.048 & 17.584 & 1.028 & 300.668 \\
\hline \multicolumn{5}{|c|}{ ER visit or readmit 90 days, patients who received telephone assessment $(n=245)$} \\
\hline Length of stay, days & 0.038 & 1.085 & 1.005 & 1.173 \\
\hline Discharge with chest tube & 0.001 & 5.685 & 2.081 & 15.529 \\
\hline Major issue on phone assessment & 0.056 & 2.603 & 0.978 & 6.929 \\
\hline
\end{tabular}

readmission. Patients with risk factors for ER visit or readmission such as pulmonary complication during initial hospitalization and those discharged home with chest tube are two patient populations that could be targeted with these protocols. Improvements could be made in the availability for seeing patients in clinic, especially after identifying issues on telephone assessments. These developments could decrease hospital readmission rates after pulmonary resection.

Pulmonary complications appear to be a significant factor in hospital readmission in our study and several others $(1,8)$. Enhanced recovery after surgery (ERAS) protocols have recently shown to be associated with decreased pulmonary complications after pulmonary resection $(20,21)$. We recently reviewed our experience with the implementation of an ERAS protocol at the MD Anderson Cancer Center. Pulmonary complications were decreased from $29 \%$ to $20 \%(\mathrm{P}=0.004)$ for all patients and $30 \%$ to $21 \%(\mathrm{P}=0.023)$ for patients who underwent thoracotomy (20). It is logical that decreased pulmonary complications should translate into decreased hospital readmissions; however, additional work is needed to determine the impact of ERAS protocols on ER visits and readmissions (22). The present study regarding post-discharge nursing telephone assessment was performed during a transitional time period of our ERAS implementation. During this time period elements of ERAS protocol were introduced, but not routinely used by all surgeons.

Previous studies focused on pulmonary resections have identified that a significant number $(17-28 \%)$ of readmissions occur to a facility that did not perform the initial operation $(1,23)$. This is an important finding to consider when interpreting our results. We found that patients that lived within 50 miles of our facility were more likely to have a hospital readmission on univariate analysis, which substantiates the previous studies. In our study, readmissions to an outside facility were included in the reported ER visit and readmission rate. However, it is possible that our hospital readmission and ER visits rates are underestimating the true incidence. There are additional limitations to our study. It was a retrospective review of a single institution and may not be generalizable to other institutions. Not all patients received nursing telephone assessments and there may be a selection bias in patients who were able to receive nursing telephone assessment and those that were not.

ER visits and hospital readmissions were significant after pulmonary resections. Pulmonary complications during initial hospitalization were associated with readmission. Standardized nursing telephone assessments were able to identify a high-risk population likely to need ER visit or readmission. However, telephone assessments did not decrease ER visits or readmissions. Improved post-discharge protocols are needed for these high-risk patients in order to prevent postoperative ER visits and hospital readmissions. These outcomes are imperative as we consider patient safety, consumer experience, and allocation of limited resources.

\section{Acknowledgments}

Presented at the 27th European Conference on General 
Thoracic Surgery of the European Society of Thoracic Surgeons, Dublin, Ireland, 9-12 June 2019.

Funding: None.

\section{Footnote}

Conflicts of Interest: MBA serves as the unpaid editorial board member of fournal of Thoracic Disease from Aug 2019 to Jul 2021. The other authors have no other conflicts of interest to declare.

Ethical Statement: The authors are accountable for all aspects of the work in ensuring that questions related to the accuracy or integrity of any part of the work are appropriately investigated and resolved. The approval was obtained from the institutional review board (PA14-0771).

Open Access Statement: This is an Open Access article distributed in accordance with the Creative Commons Attribution-NonCommercial-NoDerivs 4.0 International License (CC BY-NC-ND 4.0), which permits the noncommercial replication and distribution of the article with the strict proviso that no changes or edits are made and the original work is properly cited (including links to both the formal publication through the relevant DOI and the license). See: https://creativecommons.org/licenses/by-nc-nd/4.0/.

\section{References}

1. Hu Y, McMurry TL, Isbell JM, et al. Readmission after lung cancer resection is associated with a 6-fold increase in 90-day postoperative mortality. J Thorac Cardiovasc Surg 2014;148:2261-7.e1.

2. Lum HD, Studenski SA, Degenholtz HB, et al. Early hospital readmission is a predictor of one-year mortality in community-dwelling older Medicare beneficiaries. J Gen Intern Med 2012;27:1467-74.

3. Tsai TC, Joynt KE, Orav EJ, et al. Variation in surgicalreadmission rates and quality of hospital care. $\mathrm{N}$ Engl J Med 2013;369:1134-42.

4. Farjah F, Wood DE, Varghese TK, et al. Health care utilization among surgically treated Medicare beneficiaries with lung cancer. Ann Thorac Surg 2009;88:1749-56.

5. Freeman RK. Hospital Readmission Following Thoracic Surgery. Thorac Surg Clin 2017;27:315-26.

6. Benbassat J, Taragin M. Hospital readmissions as a measure of quality of health care: advantages and limitations. Arch Intern Med 2000;160:1074-81.
7. Rajaram R, Ju MH, Bilimoria KY, et al. National evaluation of hospital readmission after pulmonary resection. J Thorac Cardiovasc Surg 2015;150:1508-14.e2.

8. Stiles BM, Poon A, Giambrone GP, et al. Incidence and Factors Associated With Hospital Readmission After Pulmonary Lobectomy. Ann Thorac Surg 2016;101:43442; diacussion 442-3.

9. Varela G, Aranda JL, Jimenez MF, et al. Emergency hospital readmission after major lung resection: prevalence and related variables. Eur J Cardiothorac Surg 2004;26:494-7.

10. McDevitt J, Kelly M, Comber H, et al. A population-based study of hospital length of stay and emergency readmission following surgery for non-small-cell lung cancer. Eur J Cardiothorac Surg 2013;44:e253-9.

11. Dickinson KJ, Taswell JB, Allen MS, et al. Unplanned Readmission After Lung Resection: Complete Follow-Up in a 1-Year Cohort With Identification of Associated Risk Factors. Ann Thorac Surg 2017;103:1084-91.

12. Ibrahim AM, Nathan H, Thumma JR, et al. Impact of the Hospital Readmission Reduction Program on Surgical Readmissions Among Medicare Beneficiaries. Ann Surg 2017;266:617-24.

13. Desai NR, Ross JS, Kwon JY, et al. Association Between Hospital Penalty Status Under the Hospital Readmission Reduction Program and Readmission Rates for Target and Nontarget Conditions. JAMA 2016;316:2647-56.

14. Sayin Y, Kanan N. Reasons for nursing telephone counseling from individuals discharged in the early postoperative period after breast surgery. Nurs Forum 2010;45:87-96.

15. Harrison JD, Auerbach AD, Quinn K, et al. Assessing the impact of nurse post-discharge telephone calls on 30-day hospital readmission rates. J Gen Intern Med 2014;29:1519-25.

16. Mistiaen $\mathrm{P}$, Poot E. Telephone follow-up, initiated by a hospital-based health professional, for postdischarge problems in patients discharged from hospital to home. Cochrane Database Syst Rev 2006;(4):CD004510.

17. Balaban RB, Weissman JS, Samuel PA, et al. Redefining and redesigning hospital discharge to enhance patient care: a randomized controlled study. J Gen Intern Med 2008;23:1228-33.

18. Dudas V, Bookwalter T, Kerr KM, et al. The impact of follow-up telephone calls to patients after hospitalization. Am J Med 2001;111:26S-30S.

19. Antonoff MB, Ragalie W, Correa AM, et al. Results of Postdischarge Nursing Telephone Assessments: Persistent 
Symptoms Common Among Pulmonary Resection

Patients. Ann Thorac Surg 2016;102:276-81.

20. Van Haren RM, Mehran RJ, Mena GE, et al.

Enhanced Recovery Decreases Pulmonary and Cardiac

Complications After Thoracotomy for Lung Cancer. Ann

Thorac Surg 2018;106:272-9.

21. Martin LW, Sarosiek BM, Harrison MA, et al.

Implementing a Thoracic Enhanced Recovery Program:

Lessons Learned in the First Year. Ann Thorac Surg

Cite this article as: Van Haren RM, Correa AM, Sepesi B, Rice DC, Hofstetter WL, Roth JA, Swisher SG, Walsh GL, Vaporciyan AA, Mehran RJ, Antonoff MB. Hospital readmissions after pulmonary resection: post-discharge nursing telephone assessment identifies high risk patients. J Thorac Dis 2020;12(3):184-190. doi: 10.21037/jtd.2020.02.08
2018;105:1597-604.

22. Van Haren RM, Atay SM. Enhancing the study of enhanced recovery after thoracic surgery: methodology and population-based approaches for the future. J Thorac Dis 2019;11:S612-8.

23. Handy JR Jr, Child AI, Grunkemeier GL, et al. Hospital readmission after pulmonary resection: prevalence, patterns, and predisposing characteristics. Ann Thorac Surg 2001;72:1855-9; discussion 1859-60. 